\title{
Numerical study of the particle sedimentation in a viscous fluid using a coupled DEM-IB-CLBM approach
}

\author{
Ya Zhang, ${ }^{\mathrm{a}, \mathrm{b}}$, Yonghao Zhang ${ }^{\mathrm{b}}$, Guang Pan ${ }^{\mathrm{a}}$, Sina Haeri ${ }^{\mathrm{b}, *}$ \\ ${ }^{a}$ School of Marine Science and Technology, Northwestern Polytechnical University, \\ Xian, 710072, China \\ ${ }^{b}$ James Weir Building, Department of Mechanical and Aerospace Engineering, University of \\ Strathclyde, Glasgow, UK, G1 1 XJ
}

\begin{abstract}
At low terminal Reynolds numbers $R e=2-10$, there are multiple asymmetrical principal movement states for the sedimentation of a pair of particles in a channel filled with a viscous fluid. The main emphasis of this work is to investigate the formation process of these states and the effect of the natural rotation on the process when particles are released symmetrically. The flow field around each particle is fully resolved with an Immersed Boundary Method (IBM) coupled with the Cascaded Lattice Boltzmann Method (CLBM). An improved algorithm is developed to couple IBM with CLBM which can fully exploit the Graphic Processing Unit (GPU) for parallelisation. The collision between particles is handled with the Discrete Element Model (DEM). The approach is validated considering the sedimentation of a single particle released asymmetrically and also the Drafting-Kissing-Tumbling (DKT) problem of two particles.

The trajectories of particles corresponding to different principal movement states are determined for the sedimentation of a pair of particles released symmetrically in a long narrow channel. By analysing the trajectories, it is found that particles go through two distinct symmetry breaking phenomena, a sudden lateral migration that leads to asymmetrical movement centres, and (or) a
\end{abstract}

\footnotetext{
* Corresponding author

Email address: sina.haeri@strath.ac.uk (Sina Haeri )
}

Preprint submitted to Journal of Computational Physics

May 6, 2018 
divergent oscillation that leads to a zero phase lag between the fundamental frequencies of oscillating particles. Since particle's lateral movement and natural rotation display a strong coherence of nearly 1.0 over a transitional oscillatory period, the trajectories of particles without rotational degree-of-freedom are then considered to determine the impacts of natural rotation on the principal movement states. It is shown that the lateral migration can still take place even after removing the rotational degree-of-freedom. However, the divergent oscillation disappears, which makes particles move in a steady oblique or horizontal structure and leads to a smaller terminal Reynolds number.

Keywords: Particle sedimentation, Immersed Boundary, Fully Resolved Simulations, Cascaded Lattice Boltzmann, Co-processor Acceleration, Graphics Processing Unit

2010 MSC: 00-01, 99-00

\section{Introduction}

The multi-particle sedimentation in a narrow channel filled with a viscous fluid is a very complex problem, which involves the hydrodynamic and repulsive forces from the walls in addition to the contact force between particles [1, 2].

5 The analysis of this problem requires a computational method that can capture the dynamics accurately. For this reason a Cascaded Lattice Boltzmann Method (CLBM) in conjunction to Fully Resolved Simulations (FRS) of particulate flows is adopted in this study.

The lattice Boltzmann method (LBM) has a great advantage in dealing with large-scale simulations on parallel systems due to its simplicity and computational locality [3]. Thus, it is very attractive to adopt the LBM as the fluid field solver for fully resolved simulations of particulate flows and in particular, very efficient implementation on Graphical Processing Units (GPU) could be achieved. In addition, it has been proven that the Navier-Stokes equations can be recovered with second-order accuracy in the Chapman-Enskog expansion analysis [4. Therefore, the LBM method has been used widely as an efficient 
fluid field solver for fluid-solid interaction problems [1, 3, 5], 8] as an alternative to conventional Computational Fluid Dynamics techniques based on the Navier-Stokes equations.

Several LBM schemes [3] have been proposed in the literature. However, the Cascaded Lattice Boltzmann Method (CLBM) of Geier et al. [9], considerably improves the numerical stability of LB schemes while preserving the accuracy and simplicity of LBM [10 12. The improvement of stability results from the removal of the "ghost modes" [10, 11 and by performing collisions in a moving 25 reference frame, the scheme naturally achieves a higher degree of Galilean invariance [13. Improvements have also been suggested for recovery of Galilean invariance in lattice Boltzmann models [14, 15.

Premnath and Banerjee [13] proposed and derived source terms in the CLBM to represent the effect of external or internal forces on the dynamics of fluid mo30 tion. However, alternative formulation to incorporate forcing term to improve accuracy and convergence was later proposed [16, 17. To accelerate low-Mach steady-state convergence, Hajabdollahi and Premnath 18 developed a simple and efficient preconditioned formulation of the CLBM by modifying the equilibrium moments and source moments. The CLBM has also been extended to 35 shallow water equations [19] and was validated against analytical and experimental cases. Geier et al. [20, 21] proposed a parametrisation of the CLBM for fourth order accurate diffusion, which gives a good framework for high Reynolds number problems.

For engineering applications, to consider the particle motion in the fluid, low40 fidelity modelling techniques may be used [22]. The Fully Resolved Simulations (FRS) on the other hand, use a high grid resolution to determine the flow field around individual particles. Therefore, despite the high computational costs, FRS offer an accurate framework to investigate the fundamental physical mechanisms of fluid-particle interactions which are not achievable with any other 45 simulation technique. In a fully resolved approach, a fine grid is required around each particle to determine the flow-field and since the particles move, re-meshing is required if a conventional body conformal grid is adopted. To overcome the 
re-meshing difficulties of complex physical domains, fixed mesh approaches are commonly used as an alternative, see Haeri and Shrimpton 23. for a detailed review. Several approaches exist, such as the Fictitious Domain Methods (FDM) [24, 25] and the Immersed Boundary Methods (IBM) [26. In IBM, the no-slip boundary condition is enforced by adding a body force term to the underlying equations (the Navier-Stokes or the Lattice-Boltzmann) at predefined boundary points. In this paper a coupled IB-CLBM approach has been implemented. The method is similar to IB-CLBM approach proposed by De Rosis and Lévêque [27. who demonstrated its flexibility, stability, and accuracy using various fluid-solid interaction problems.

In IBM it is vital to find an accurate forcing scheme to satisfy the no-slip condition on the surface of solid particles. In this paper, the Multi Direct 60 Forcing Method (MDFM) proposed by Wang et al. 28] and later coupled with LBM by Suzuki and Inamuro 29] is employed, which improves accuracy of the no-slip boundary condition by solving the body force iteratively. It has been validated with experimental and theoretical results 29, 30, and was used in the simulation of butterfly-like and dragonfly-like flapping wings [31].

In simulations involving multiple particles, a reliable collision strategy is also required to accurately model the transfer of kinetic energy and also to avoid overlaps. The Discrete Element Model (DEM) is perhaps the most reliable approach with a sound theoretical foundation to resolve particle collision. DEM has been applied to a wide range of problems involving particles spanning from dry granular systems 32,33 to particulate flows at laminar to turbulent regimes 34. In the present paper a coupled DEM-IB-CLBM approach has been developed and implemented on a single GPU and the reader is referred to Appendix A for further information regarding the speed-up and implementation details.

The sedimentation of a pair of particles inside a narrow vertical channel exhibits a variety of regimes due to strong hydrodynamic interactions between particles [1, 2, 7. Here, the terminal Reynolds number $R e_{t}$, is defined as $R e_{t}=$ $U_{t} D / \nu$, where $U_{t}, D$ and $\nu$ are the average particle's terminal velocity, the 
particle diameter and the kinematic viscosity respectively. Feng et al. [7] found that the two particles would form an oblique doublet structure or settle with a repetitive Drafting-Kissing-Tumbling (DKT) motion. Aidun and Ding [1] investigated several cases with $2<R e_{t}<6$ and discovered a period-doubling state. After expanding the range of $R e_{t}$ to 10, Verjus et al. 2] found a quasiperiodic state and its leading chaotic attractor. They also established a global diagram of the terminal Reynolds number versus the non-dimensional driving force and indicated there could be more movement states for a larger Reynolds number.

So far, most of the numerical studies focus on global quantities such as the principal movement states (the final stationary state of particle motion) and 90 terminal settling velocities. However, the literature on the formation process of the principal movement states is sparse and the mechanisms are not well understood. A pair of particles released symmetrically will experience a transition from symmetrical to asymmetrical sedimentation to form the principal movement states. Feng et al. 7] investigated this problem by performing simulations on the symmetric particle pair at $R e_{t}=1.52$ with a channel confinement of 8 and identified a lateral migration during the transition to an asymmetric state. Contrary to Feng's findings, Jayaweera and Mason 35] did not mention this movement in their similar experiments. Therefore, further investigation is required to pinpoint the mechanism leading to the formation of principal movement states.

Moreover, the Magnus force induced by particle rotation is perpendicular to the velocity and the axis of rotation which can make a spinning particle curve away from its principal moving path. For sedimentation in a two-dimensional channel, where particles keep moving downward, the Magnus force induced by a natural rotation, mainly acts on the lateral movement of particles, and could greatly influence their trajectories and even the principal states. In this work, the effects of particle's natural rotation will also be considered to further investigate the formation process of the principal movement states.

The rest of the paper is organized as follows: In Section 2 the DEM-IB- 
CLBM approach is briefly discussed and a new improved algorithm is presented. The DEM-IB-CLBM implementation is validated in Section 3 by considering the sedimentation of a single particle and the DKT problem. In Section 4 , the sedimentation of a pair of particles released symmetrically is investigated to obtain the particle trajectories corresponding to several typical principal movement states. In Section 5, the formation process of these states and the effect of particle's natural rotation on the process are analysed in detail and concluding remarks are presented in Section 6 .

\section{Numerical Methods}

\subsection{Cascaded Lattice Boltzmann Method (CLBM)}

In the CLMB, the fluid is described as imaginary particles, which stream along a uniform lattice grid at the fixed reference frame and collide with each other at the moving reference frame. The particles are modelled with a set of distribution functions which evolve according to the following equation:

$$
\mathbf{f}(\mathbf{x}+\mathbf{e} \delta t, t+\delta t)=\mathbf{f}(\mathbf{x}, t)+\boldsymbol{\Omega}(\mathbf{x}, t)+\mathbf{S} \delta t,
$$

where $\mathbf{f}$ is the vector of the particle distribution functions representing the probability of finding a particle at the position $\mathbf{x}$ with the discrete velocity $\mathbf{e}$ at the time $t ; \boldsymbol{\Omega}, \mathbf{S}$ and $\delta t$ are the vector of the discrete collision operators, the vector of the discrete force terms and the time step respectively.

The nine-velocity square lattice model for two-dimensional flows (D2Q9) [36] is used in this work. The discrete velocity set $\mathbf{e}$ is sketched in Fig, 1 .

$$
\mathbf{e}=\left[\begin{array}{rrrrrrrrr}
0 & 1 & 0 & -1 & 0 & 1 & -1 & -1 & 1 \\
0 & 0 & 1 & 0 & -1 & 1 & 1 & -1 & -1
\end{array}\right] .
$$

Premnath and Banerjee [13 incorporated the force term in the centralmoment collision operators and derived analytical expressions for the discrete forces and their moments. The collision operators are obtained from $\boldsymbol{\Omega}=\mathbf{K} \cdot \mathbf{k}$, where $\mathbf{K}$ is a transformation matrix and $\mathbf{k}$ is the vector of the moments of the 
distribution functions $\mathbf{f}$, cf. [13. The discrete forcing term $\mathbf{S}$ is obtained from the body force $\mathbf{F}=\left(F_{x}, F_{y}\right)[13$. To maintain the isotropy of the stress tensor, the kinematic shear viscosity is connected to the relaxation parameters $\omega_{\alpha}$ as follows

$$
\nu=c_{s}^{2}\left(\omega_{4,5}^{-1}-0.5\right),
$$

$$
\omega_{4}=\omega_{5},
$$

where other relaxation parameters are set to unity, and $c_{s}$ is the sound speed of the D2Q9 model, $c_{s}=1 / \sqrt{3}$. The Mach number $M a=u / c_{s}$ should be small to limit the compressibility errors in the LBM, so the fluid velocity in lattice units is keeping lower than 0.05 . The macroscopic density $\rho$ and velocities $\mathbf{u}=\left(u_{x}, u_{y}\right)$ can be obtained by taking the zeroth and first moments of the distribution functions as follows

$$
\begin{gathered}
\rho=\sum_{\alpha=0}^{8} f_{\alpha}, \\
\rho \mathbf{u}=\sum_{\alpha=0}^{8} \mathbf{e}_{\alpha} f_{\alpha}+\frac{1}{2} \mathbf{F} \delta t .
\end{gathered}
$$

\subsection{Immersed boundary method}

To simulate the particles in a fluid the IBM is adopted. The no-slip boundary condition is enforced with an appropriate body force at the fluid nodes near the solid particle boundaries. The computational domain is discretized using a fixed Eulerian mesh and the surface of solid particles is represented by a moving Lagrangian grid. The two grids communicate with each other through an interpolation/distribution scheme which will be described next. The variables in the Lagrangian frame are represented with a subscript $l$ and the variables in the Eulerian frame have no subscript. The fluid velocity at the Lagrangian points $\mathbf{x}_{l}$ is interpolated from the surrounding lattices using

$$
\mathbf{u}\left(\mathbf{x}_{l}\right)=\sum_{\mathbf{x}} \mathbf{u}^{*}(\mathbf{x}) \Phi\left(\mathbf{x}-\mathbf{x}_{l}\right) \delta x^{2},
$$


where $\delta x$ is the lattice spacing and $\mathbf{u}^{*}$ is the fluid velocity vector calculated as

$$
\mathbf{u}^{*}=\frac{1}{\rho} \sum_{\alpha=0}^{8} \mathbf{e}_{\alpha} f_{\alpha} .
$$

The discrete delta function $\Phi$ can be expressed as

$$
\Phi(x, y)=\frac{1}{\delta x^{2}} \phi\left(\frac{x}{\delta x}\right) \phi\left(\frac{y}{\delta x}\right) .
$$

Haeri and Shrimpton 23. have tested the accuracy of several different delta functions for application to many particle systems and showed that 3 and 4point regularized delta functions provide a good trade-off between accuracy and computational costs. In this paper the following 4-point regularised delta function is used

$$
\phi(r)= \begin{cases}\frac{1}{8}\left(3-2|r|+\sqrt{1+4|r|-4 r^{2}}\right), & |r| \leq 1 \\ \frac{1}{8}\left(5-2|r|-\sqrt{-7+12|r|-4 r^{2}}\right), & 1 \leq|r| \leq 2 \\ 0, & \text { otherwise. }\end{cases}
$$

The velocity corrections to the IB points are obtained as follows

$$
\delta \mathbf{u}_{l}\left(\mathbf{x}_{l}\right)=\left(\mathbf{u}_{l}\left(\mathbf{x}_{l}\right)-\mathbf{u}\left(\mathbf{x}_{l}\right)\right) \delta x,
$$

and are redistributed onto the background Eulerian grid by

$$
\delta \mathbf{u}(\mathbf{x})=\sum_{\mathbf{x}_{l}} \delta \mathbf{u}_{l}\left(\mathbf{x}_{l}\right) \Phi\left(\mathbf{x}-\mathbf{x}_{l}\right) \delta s
$$

In Eq. 12,$\delta s$ is the spacing of the Lagrangian grid used to represent the solid boundaries. The body forces on the fluid nodes $\mathbf{F}^{i b}(\mathbf{x})$ and the IB points $\mathbf{F}_{l}^{i b}\left(\mathbf{x}_{l}\right)$ can be obtained by

$$
\mathbf{F}^{i b}(\mathbf{x})=\frac{2 \rho \delta \mathbf{u}(\mathbf{x})}{\delta t}
$$

and

$$
\mathbf{F}_{l}^{i b}\left(\mathbf{x}_{l}\right)=\frac{2 \rho \delta \mathbf{u}_{l}\left(\mathbf{x}_{l}\right)}{\delta t}
$$

respectively, which is commonly referred to as a Direct Forcing Method. However, the method does not accurately satisfy the no-slip boundary conditions. To 
solve this problem, Wu and Shu [37] suggested an implicit correction. However, there is a huge computational overhead due to required matrix inversions, which makes it infeasible for simulations involving multiple moving particles. Suzuki and Inamuro 29] suggested the MDFM that avoids the matrix inversion.

However, it should be noted that the force scheme used by Suzuki and Inamuro [29] is substantially different from that of the CLBM scheme. The former takes into account the body force only in the collision procedure, while the latter uses a split-forcing model similar to the force scheme proposed by Guo et al. 38], which also redefines the velocity to present the force density implicitly. Therefore, a new MDFM algorithm is developed here to enable its efficient coupling with the CLBM. The algorithm can be summarised as follows:

1. Set $\mathbf{f}^{n}$ to $\mathbf{f}^{\prime n-1}$, where $\mathbf{f}^{\prime}$ is the post-collision distribution function;

2. Calculate $\rho^{n}(\mathbf{x})$ and $\mathbf{u}^{* n}(\mathbf{x})$ using Eq. (5) and Eq.(8), respectively;

3. The body force to enforce the no-slip condition is then calculated through the following iterative scheme: ( $i$ is the loop counter and $N$ is the maximum number of iterations allowed);

(a) Initialize the velocity corrections for the IB points with $\delta \mathbf{u}_{l}\left(\mathbf{x}_{l}\right)=0$;

(b) Start the iterative process and set $i$ to 0 ;

(c) Interpolate the fluid velocity at Lagrangian points $\mathbf{u}^{i}\left(\mathbf{x}_{l}\right)$ using Eq. (7);

(d) Obtain the $i^{\text {th }}$ velocity corrections to IB points $\delta \mathbf{u}_{l}^{i}\left(\mathbf{x}_{l}\right)$ from Eq. 111, and add the corrections to $\delta \mathbf{u}_{l}\left(\mathbf{x}_{l}\right)$;

(e) Calculate the $i^{\text {th }}$ velocity corrections to fluid grids $\delta \mathbf{u}^{i}(\mathbf{x})$ using Eq. 12, and add the corrections to the fluid velocity $\mathbf{u}^{* n}(\mathbf{x})$;

(f) Increment $i$ and repeat (c) to (e) while $i \leq N$;

(g) Set $\mathbf{u}^{n}(\mathbf{x})$ to $\mathbf{u}^{* n}(\mathbf{x})$.

(h) Compute the IB force $\mathbf{F}_{l}^{i b}\left(\mathbf{x}_{l}\right)$ using Eq. (14);

(i) Compute the fluid force $\mathbf{F}^{i b}(\mathbf{x})$ using Eq.12 and Eq. 13). Set $\mathbf{F}^{n}(\mathbf{x})$ to $\mathbf{F}^{i b}(\mathbf{x})$ to be used as the body forcing term in Eq.(1) and Eq.(6).

4. Set $\mathbf{f}^{\prime n}$ to $\mathbf{f}^{n}+\boldsymbol{\Omega}^{n}+\mathbf{S}^{n} \delta t$, where $\boldsymbol{\Omega}^{n}$ and $\mathbf{S}^{n}$ are obtained from $\rho^{n}(\mathbf{x})$, $\mathbf{u}^{n}(\mathbf{x})$, and $\mathbf{F}^{n}(\mathbf{x})$. 
In this new algorithm the fluid velocity $\mathbf{u}^{n}(\mathbf{x})$ is calculated cumulatively, which saves the computational resources. This is due to the fact that if $\mathbf{u}^{n}(\mathbf{x})$ were calculated using Eq. (6) similar to previously suggested algorithm [27, then a copy of $\mathbf{u}^{* n}(\mathbf{x})$ would have to be stored before the iteration for interpolations. Considering the limited size of the GPU memory, this is particularly important for implementation on a GPU. In addition, a similar cumulative correction scheme has been suggested by Haeri and Shrimpton [24, 39] for their Fictitious Domain Methods in conjunction with a Finite Volume (FV) NavierStokes solver.

The number of loops $N$ is set to 5 , enough to accurately satisfy the no-slip condition [29, 30]. The Lagrangian mesh spacing $\delta s$ is chosen close to $\delta x$ and within the range of $(0.5 \delta x, \delta x)$ to avoid a fluid flux through the solid boundaries while no unnecessary computational overhead is inflicted due to an excessively dense meshing [40.

The hydrodynamic force and torque acting on the particle are given by

$$
\begin{gathered}
\mathbf{F}_{h}=-\sum_{\mathbf{x}_{l}} \mathbf{F}_{l}^{i b}\left(\mathbf{x}_{l}\right) \delta s+\mathbf{F}_{i n}, \\
\mathbf{T}_{h}=-\sum_{\mathbf{x}_{l}}\left(\mathbf{x}_{l}-\mathbf{X}\right) \times \mathbf{F}_{l}^{i b}\left(\mathbf{x}_{l}\right) \delta s+\mathbf{T}_{i n},
\end{gathered}
$$

where $\mathbf{X}$ is the coordinates of the particle centre, $\mathbf{F}_{i n}$ and $\mathbf{T}_{i n}$ are the force and torque resulting from the movement of fluid inside the immersed surface. To calculate $\mathbf{F}_{\text {in }}$ and $\mathbf{T}_{\text {in }}$ Feng's rigid body approximation [29, 41] is used:

$$
\begin{gathered}
\mathbf{F}_{i n}^{n}=\frac{\rho_{f}}{\rho_{p}} m_{p} \frac{\mathbf{U}^{n}-\mathbf{U}^{n-1}}{\delta t}, \\
\mathbf{T}_{i n}^{n}=\frac{\rho_{f}}{\rho_{p}} I_{p} \frac{\boldsymbol{\Omega}_{c}^{n}-\boldsymbol{\Omega}_{c}^{n-1}}{\delta t},
\end{gathered}
$$

where $\rho_{f}, \rho_{p}, m_{p}$ and $I_{p}$ are respectively, the fluid and particle densities, the mass and moment of inertia of the particle. $\mathbf{U}$ and $\boldsymbol{\Omega}_{c}$ are the translational and angular velocities of the particle's centre of mass, which is then used to update the location and velocity of the Lagrangian points on the particle surface using rigid body dynamics, see Haeri and Shrimpton 24] for more details. 


\subsection{Discrete Element Method}

To consider particle collisions, a soft-sphere approach is used which allows particles to overlap. The contact force is modelled as a function of the overlapping thickness and the relative particle velocities.

In the present work, we resort to a linear spring-dashpot model [42, to deal with the particle-particle and particle-wall collisions when the gap is less than a critical value $\epsilon_{c}$. The overlap length is given by $\delta_{n}=\left(R_{i}+R_{j}+\epsilon_{c}\right)-\left|\mathbf{X}_{i}-\mathbf{X}_{j}\right|$, where $R$ is the radius of a pair of particles $i, j$. The normal and tangential contact forces are calculated from

$$
\begin{gathered}
F_{c n}=k_{n} \delta_{n}+c_{n} v_{r n}, \\
F_{c t}= \begin{cases}F_{c t}^{o l d}+k_{t} \Delta \delta_{t}+c_{t} v_{r t}, & F_{c t}<\mu F_{c n} \\
\mu F_{c n}, & F_{c t} \geq \mu F_{c n}\end{cases}
\end{gathered}
$$

where $k_{n}, k_{t}$ and $c_{n}, c_{t}$, are the normal and tangential spring and damping coefficients, respectively. The total relative velocity between the particle surfaces at contact point is $\mathbf{V}_{r}=\mathbf{U}_{i}-\mathbf{U}_{j}+R_{i} \mathbf{n} \times \boldsymbol{\Omega}_{c i}+R_{j} \mathbf{n} \times \boldsymbol{\Omega}_{c j}$. The relative velocity in normal direction is $v_{r n}=-\mathbf{V}_{r} \cdot \mathbf{n}$ and the relative tangential velocity at the contact point is $v_{r t}=-\mathbf{n} \times\left(\mathbf{V}_{r}-v_{r n} \mathbf{n}\right)$. The unit normal vector is $\mathbf{n}=\left(\mathbf{X}_{i}-\mathbf{X}_{j}\right) /\left|\mathbf{X}_{i}-\mathbf{X}_{j}\right|$, and the unit tangential vector is $\mathbf{t}=\left(-n_{y}, n_{x}\right) . \Delta \delta_{t}$ is the increment of the tangential relative surface displacement. The particlewall collisions are handled in the similar way, where $\delta_{n}=\left(R_{i}+\epsilon_{c}\right)-\left|\mathbf{X}_{i}-\mathbf{X}_{\text {wall }}\right|$, $\mathbf{U}_{\text {wall }}=0$ and $\omega_{\text {wall }}=0$. Then the total contact force exerted on a particle $i$ by other particles and walls is

$$
\mathbf{F}_{c}=\sum^{p, \text { wall }}\left(F_{c n} \mathbf{n}+F_{c t} \mathbf{t}\right)
$$

In this case, we consider the collisions between particles with low relative velocities. Therefore, we only consider the normal forces by setting the $k_{t}=0$ and $c_{t}=0$. Several normal forcing strategies have been suggested before [25] but the current DEM based approach is more accurate and is physically well 
defined, see Haeri [32, Haeri et al. 33. for a detailed discussion on the linear spring-dashpot model. In the present immersed boundary method, $\epsilon_{c}$ is set to $2 \delta x$ [24, 25], which is equivalent to the range of adopted 4-point regularized delta function. The normal stiffness parameter, is chosen such that the forcing term is equivalent to the available DKT benchmark problems, see Section 3.2 for more details.

Each particle's centre of mass is governed by the Newton's second law of motion:

$$
\left\{\begin{array}{l}
\frac{d^{2} \mathbf{X}}{d t^{2}}=\mathbf{F}_{p} / m_{p} \\
\frac{d^{2} \boldsymbol{\Theta}}{d t^{2}}=\mathbf{T}_{p} / I_{p} \\
\frac{d \mathbf{X}}{d t}=\mathbf{U} \\
\frac{d \boldsymbol{\Theta}}{d t}=\boldsymbol{\Omega}_{c}
\end{array}\right.
$$

where $\mathbf{F}_{p}$ is the sum of the buoyancy, contact forces and hydrodynamic force from fluid-particle interaction, $\mathbf{F}_{p}=\left(\frac{\rho_{f}}{\rho_{p}}-1\right) m_{p} \mathbf{g}+\mathbf{F}_{c}+\mathbf{F}_{h}$ and $\mathbf{T}_{p}$ is the total torque $\left(\mathbf{T}_{p}=\mathbf{T}_{h}\right)$. The angular displacement around the centre of the particle is represented by $\boldsymbol{\Theta}$. A second order integration scheme is used to integrate the set of Eq. 22 as follows:

$$
\left\{\begin{array}{l}
\mathbf{X}^{n}=2 \mathbf{X}^{n-1}-\mathbf{X}^{n-2}+\frac{\mathbf{F}_{p}^{n-1}}{m_{p}} \delta t^{2}, \\
\boldsymbol{\Theta}^{n}=2 \Theta^{n-1}-\Theta^{n-2}+\frac{\mathbf{T}_{p}^{n-1}}{I_{p}} \delta t^{2}, \\
\mathbf{U}^{n}=\frac{\mathbf{X}^{n}-\mathbf{X}^{n-2}}{2 \delta t} \\
\boldsymbol{\Omega}_{c}^{n}=\frac{\Theta^{n}-\Theta^{n-2}}{2 \delta t} .
\end{array}\right.
$$

\section{Validation of the method}

In this section, we examine the accuracy of the DEM-IB-CLBM approach by applying the method to two benchmark problems: the sedimentation of a single particle and the DKT of two particles. 
In Fig 3, particles released from different initial positions move gradually to the centreline. The settling trajectories are found to match closely with the available data [7. 


\subsection{The classical Drafting-Kissing-Tumbling}

The second test case is the classical DKT problem of two particles in a cavity. diameter $D=25 \delta x$ is used in the following simulations to save the computational resources. The vorticity contours of the two particles during the collision is depicted in Fig 6 


\section{The sedimentation of a pair of particles}

250 released symmetrically in a long narrow channel to obtain the particle trajectories corresponding to several typical principal states. The characteristics of the DEM-IB-CLBM approach is analysed for the simulations of this sensitive non-linear fluid-particle interaction problem. physical diameter $D$ while keeping the other parameters (including the confinement) unchanged according to Eq. 244. The origin of coordinate is moved to the centreline, and variables are normalised using the cylinder diameter $D$ and the kinematic viscosity $\nu$, according to $x / D, y / D, U_{r}=u_{x} D / \nu, V_{r}=u_{y} D / \nu$ and $\omega_{r}=\omega_{p} D^{2} / \nu$, which are respectively the non-dimensional coordinates, translational and angular velocities.

Initially, particles are aligned horizontally and symmetrically concerning the centreline as shown in Fig 7 and released from rest. The symmetry suggests that forces acting on the two particles are balanced, so the particles should fall symmetrically. The particle released from the left side is Particle 1 and the other one is Particle 2.

\subsection{Numerical simulations}

For a pair of particles falling in a narrow channel $\left(R e_{t}=2-10\right)$, there are four different principal movement states [1, 2]: steady oblique doublet, period doubling, DKT, and quasi-periodic as shown in Fig 8 . The overlap between the first two states is called the multi-stable stage, where particles may enter either of the two states. 
In Fig 8 , the terminal Reynolds numbers of DKT agree well with the results from Verjus et al. [2], and a period of the repeating DKT $(F=300)$ are recorded in Fig.9. There is a discrepancy for $F=130$ and $F=150$ (Steady Oblique), $F=160$ (period doubling) and $F=450$ and $F=480$ (quasi-periodic) regions. The reason is existence of a diffuse boundary with a thickness greater that a lattice in our immersed boundary method. This makes the effective diameter larger than the intended size and hence, the channel confinement is slightly smaller than 4 in the simulations.

Simulations on a finer mesh with $\delta x=D / 50$ are conducted here to verify the aforementioned hypothesis. The results are presented in Fig, 8 , which shows that the new simulations with $\delta x=D / 50$ agree better with results reported in [2]. It is interesting to note that as $F=150$ is located at the border of multi-stable range, and hence when a finer resolution is used, the movement state shifts from steady oblique doublet to period doubling state. In Fig 10 , the phase portrait of $F=480$ with $D=50 \delta x$ is compared with the corresponding results in 2 illustrating an excellent agreement.

The phase portraits of $F=450$ and $F=480$ with $D=25 \delta x$ are plotted in 295 Fig 11 . They show similar characteristics compared to $F=480$ and $F=489.5$ reported in [2], respectively. It demonstrates that a smaller confinement caused by the boundary thickness forces the states to appear earlier than expected. Therefore, if the problem is susceptible or there is a strict requirement for the confinement size, the DEM-IB-CLBM approach should be used with care. The influence of diffuse boundary could be reduced by increasing the fluid field resolution or considering an effective boundary thickness 30. In this work, however, we focus on the particle trajectories that correspond to the principal movement states, which have been captured successfully with the resolution $D=25 \delta x$, thus the following analysis is still based on the results from particles with $D=25 \delta x$. 


\section{Results and Discussions}

This section is concerned with the formation process of the principal movement states based on the trajectories of particles obtained in the simulations. The characteristics of the formation process of the principal movement states are determined using a time-frequency analysis.

\subsection{The formation process of the principal movement states}

Fig 12 shows the trajectories of particle pairs corresponding to different principal movement states. It seems that particles experience dramatically different trajectories to reach their principal movement states.

Fig 12 a shows the motion of particles with $F=150$. Applying a small initial disturbance (which will be discussed in detail later in this section), a sudden lateral migration of both particles to the right wall breaks the symmetrically moving centres and leads to a steady oblique structure. Fig 13 represents more details of the lateral and angular velocities of the pair of particles. After being released from Point M and N (see Fig 7 ), particles move symmetrically and rotate with a changing angular velocity in the opposite directions. The particles then come to a temporary state (Points A and B in Fig 13) where the angular and lateral velocities are nearly zero and the particles continue to settle downward. Then they experience a sudden lateral migration to the right wall after which, they settle steadily with the same terminal velocity and negative angular velocities.

Fig $12 \mathrm{~b}$ and $12 \mathrm{c}$ show the trajectories of particle with $F=160$ and $F=300$, where the particles experience a similar lateral migration to the walls. However, different from Fig $12 \mathrm{a}$, particles do not settle steadily after the migration but begin to oscillate (divergent oscillation). The amplitude and frequency of the oscillations are different for the two particles at $F=160$ but by increasing $F$ the oscillations seem to become more correlated.

Fig $12 \mathrm{~d}$ and $12 \mathrm{e}$ show the trajectories of particles with $F=450$ and $F=480$ where only the divergent oscillation that leads to the terminal quasi-periodic 
state is observed with no lateral migration. The phase portrait of this principal state is illustrated by Fig 11 , where the motion of particles'centres are nearly symmetric, and oscillate asymmetrically.

In summary, the particles experience a lateral migration and(or) a divergent oscillation, where the former leads to a steady off-centre motion and the latter leads to asymmetric oscillations. On the way to the principal movement states, the stable state $(F=150)$ only experiences the lateral migration, the quasiperiodic state $(F=450$ and $F=480)$ only experiences the divergent oscillation. The period-doubling and DKT $(F=160$ and $F=300)$ regimes can be regarded as transitional states where both phenomena occur.

The appearance of the lateral migration in Fig 12 (a)-(c) demonstrates a 345 symmetrical horizontal structure is unstable for the corresponding $F$ values. For example accumulation of numerical rounding errors may trigger the symmetry breaking. To demonstrate this the case with $F=150$ is considered. For the particles released in a perfectly symmetric arrangement (without disturbance), the particles need to travel to $y \approx 50 D$ before they experience any lateral migration. However, by introducing a small initial disturbance $(0.01 d x)$ to the vertical position of Particle 1 the lateral migration occurs earlier (at $y \approx 20 D$ ), see Fig $12 \mathrm{a}$. The particles move to the wall near Particle 1 but the size of the lateral migration is the same as before. Conversely, by adding the disturbance to Particle 2, the particles shift to the other side.

It seems that a disturbance in the motion of any one particle triggers the development of an asymmetric structure and the particles will move to the wall near the advancing particle. In experiments, the symmetry breaking could be triggered by any disturbance in the initial configuration. It is worth mentioning that if no initial disturbance is introduced the migration may take a very long time to develop and that may be the reason that a symmetric horizontal structure is often identified as another stable state. This also explains why there is no such a lateral migration in the similar experiments reported by Jayaweera and Mason 35.

The particle pairs in Fig 12(b)-(e) undergo the divergent oscillation. The 
case $F=450$ in Fig $12 \mathrm{~d}$ is considered for the following analysis of the oscillatory motion. A time-frequency analysis is used to characterise the oscillations from the point of release to the formation of the principal movement state. Fig, 14 shows the lateral velocity of Particle $1(F=450)$ along the time steps and the corresponding time-frequency analysis. Here, the relative frequency is normalized by the initial oscillation frequency when the particles start moving $\left(f_{0}=1\right)$, the power spectral density (PSD) and the lateral velocity $U_{r}$ are normalized by their maximum values, respectively.

Fig 14 illustrates three main frequencies $\left(f_{0}, f_{1}\right.$, and $\left.f_{2}\right)$ appearing on the figure. When particles are released, Particle 1 enters a single-frequency damped oscillation; between time steps $(4-6) \times 10^{5}$, two new frequencies $\left(f_{1}\right.$ and $\left.f_{2}\right)$ develop in succession with increasing amplitudes until the principal movement states form. The time step range $(4-6) \times 10^{5}$ can be regarded as a transitional period, where the initial oscillation of $f_{0}$ disappears and the new steady oscillatory motion with two new harmonics $f_{1}$ and $f_{2}$ appears.

Fig $14 \mathrm{~b}$ displays the spectrograms of the initial oscillation and the terminal oscillation, taken from the time steps marked with arrows on Fig, 14a, where the energy of the harmonic $f_{2}$ is only about $10 \%$ of that of the fundamental frequency $f_{1}$. Fig 15 gives the time-frequency coherences of the lateral and angular velocities of the Particle 1 and 2. The phase arrows displayed in the figure with a threshold of 0.5. The directions of the arrows present the phase lag of Particle 1 relative to Particle 2, here the rightward and leftwards arrows indicate a phase lag of 0 and $\pi$ respectively. A phase lag of $\pi$ indicates a symmetrical movement (around the centreline).

Fig 15 shows the analysis of particles'lateral and angular velocities. The particles initially move symmetrically with a single frequency $f_{0}$ and a phase lag $\pi$. After the transitional period $\left(t=(4-6) \times 10^{5} \Delta t\right)$, the particles move with two frequencies $f_{1}$ and $f_{2}$, where the phase lag at the fundamental frequency $f_{1}$ is 0 and the phase lag at the harmonic frequency $f_{2}$ is $\pi$. It is clear that there is a strong coherence of nearly 1.0 between the three frequencies. However, during the transitional period both coherences collapse, indicating an independent 
motion of particles.

Moreover, Fig 16 represents the coherence between the lateral and angular velocities of Particle 1. It is shown that the phase lag between the lateral and angular velocities of Particle 1 is nearly zero initially, then it grows gradually to $-\frac{\pi}{4}$ and $\frac{\pi}{4}$ at frequencies $f_{1}$ and $f_{2}$, respectively. In comparison to the weak coherence between the motion of the two particles during the transitional period, there is a significant coherence between the lateral and angular velocities of the individual particles throughout the entire settlement, see Fig 16 . The change on coherence between the lateral and rotational velocities of the particle, indicates the effects of rotation on the appearance of divergent oscillation during the transitional period.

\subsection{The effect of rotation}

From the discussions in Section 5.1, it is clear that there is a strong coherence between the lateral movement and particle's natural rotation which is interestingly maintained during the transitional period. Therefore, the effects of rotation on the particle's movement is further investigated in this section. We will explore the effect of rotation by comparing the behaviour of particles with and without the rotational degree-of-freedom. The trajectories of the particles without a rotational degree-of-freedom are presented in Fig.12.

Fig. 12(a)-(c) shows that the rotation has a little influence on the occurrence of the lateral migration despite its impact on the particles'trajectories. Fig 13 shows that $(F=150)$ the particles with natural rotation have a greater lateral velocity until they reach the temporary states at points A and B. During this period the rotation is mostly a symmetrical upward rolling against the nearest wall (i.e. positive for Particle 1 and negative for Particle 2), which induces a Magnus force pointing to the other wall. During the lateral migration to the new equilibrium positions at Points A' and B', the velocity of particles with natural rotation is still higher and they rotate in the same direction (clockwise). After reaching Points A' and B', both particle pairs with and without rotational degree-of-freedom are staggered in an oblique structure as shown in Fig 17 
However, despite the similar oblique structures, there is still a difference in the tilt angles, $20.85^{\circ}$ for the case with natural rotation, $15.12^{\circ}$ for the case without rotational degree-of-freedom. The reduction of the tilt angles leads to a larger blockage, which decreases the terminal Reynolds number to about $88.36 \%$ of that with natural rotation. Hence, we conclude that although the particle rotation is not the key to forming the oblique structure, it has a significant influence on the tilt angle and the terminal Reynolds number of particles.

Fig 12 (b)-(e) show the cases with a divergent oscillation. Here, after removing the natural rotation, at the initial symmetrical stage, the lateral velocity is damped quickly as indicated in Fig 18 . Then, the divergent oscillation vanishes, and the particles settle steadily in an oblique or a symmetric horizontal structure. The instantaneous vorticity contour of the principal movement states ( $F=300$ and $F=450)$ at two different times are presented in Fig 19 and Fig 20. Therefore, it is clear that particles'natural rotation plays a vital role in the appearance of the divergent oscillation.

The terminal Reynolds number, which usually attracts more attention in practical applications, undergoes a dramatic change after removing the rotational degree-of-freedom. Table 1 lists the values of $R e_{t}$ and the corresponding principal movement states. The various states are reduced to only the steady 445 horizontal and oblique doublet structures for the cases without a rotational degree-of-freedom. In the DKT $(F=300)$ regime $R e_{t}$ is reduced to only $42.53 \%$ of its original value, while the $R e_{t}$ of the quasi-periodic state shows less sensitivity.

\section{Conclusion}

In this paper, a DEM-IB-CLBM approach is developed and used to study the formation process of the typical principal movement states of particle pairs, which are released symmetrically and settled in a long narrow channel filled with a viscous fluid. The algorithm of IB-CLBM is improved to obtain an efficient performance particularly on the Graphic Processing Units (GPUs). The classical 

to medium Reynolds number.

The trajectories of particles corresponding to different principal movement states are calculated and the characteristics of the DEM-IB-CLBM approach is determined for this sensitive fluid-particle problem. The trajectories of particles are analysed in detail and two distinct symmetry breaking phenomena are identified, i.e. a sudden lateral migration that leads to asymmetrical movement centres, and a divergent oscillation that leads to an asymmetric oscillatory motion of the particles with a zero phase lag. Further time-frequency analysis shows a strong correlation between the particle's lateral and rotational velocities. is further investigated to pinpoint the impact of rotation on the motion of the particles. After removing the rotational degree-of-freedom, the lateral migration can still happen but particle's oscillation vanishes. Therefore, particles move in a steady oblique or horizontal structure, which cause a lower terminal Reynolds

\section{Acknowledgment}

This work is supported by the National Key Research and Development Project of China (Grant No. 2016YFC0301300); the National Natural Science Foundation of China (Grant No. 11502210 and 51479170); Ph.D.'s innovation fund of Northwestern Polytechnical University; the China Scholar Council and 480 Nvidia's GPU Grant. We would also like to thank Dr. A. De Rosis for his help on the code development. 


\section{Appendix A: Code acceleration on GPU}

In this paper, the GPU acceleration technique is employed for the parallelisation of the DEM-IB-CLBM approach. The GPU parallelisation strategy for the CLBM evolution are frequently used during the run-time and result in a significant speed-up. In the CLBM part of the code, each thread deals with one lattice so that all the lattice nodes can be updated in parallel.

For small number of particles, the movement of particle centres and treatment of the collisions (DEM) take only a small part of the computation (see 490 Haeri and Shrimpton [25] for profiling results of a typical fully-resolved solver). Nonetheless, these calculations are also off-loaded to the GPU to make it easier to extend the simulations to a large number of particles. For the DEM part each thread deals with one "physical" particle.

The MDFM (IBM model) is the bottleneck of parallel performance since it involves an iterative correction, interpolation and distribution operations. The interpolation procedure of MDFM needs to read the information of the adjacent lattices around Lagrangian points. The distribution procedure needs to distribute the velocity correction of a Lagrangian point to surrounding fluid lattices, which means corrections to a lattice come from different Lagrangian points. So there are two ways of parallel execution, the Lagrangian mesh centric arrangement and the Eulerian mesh centric arrangement [47. A Lagrangian mesh centric arrangement is adopted here for the interpolation procedure. If we take the Eulerian mesh centric arrangement, it is quite difficult for a lattice to find its nearby Lagrangian points efficiently due to the motion of particles. In the Lagrangian mesh centric arrangement an atomic operation is needed to avoid a race condition and ensure integrity of the data, although it will undermine the parallel efficiency. In this section the performance of the MFDM with a Lagrangian mesh centric arrangement on GPU is compared to its sequential counterpart on a single processor core.

For the MDFM on GPU, one thread is allocated to each Lagrangian point. The interpolation procedure in step 3.(c) of Section 2.2 can benefit most from 
GPU acceleration. The information of the adjacent lattices around a Lagrangian point is read for the interpolation. A function atomicAdd_double() with the assistance of atomicCAS() (compare and swap) is used for the distribution procedure in step 3.(e) of Section 2.2, since atomicAdd() for double-precision floating-point number is not available on devices with compute capability lower than 6.0. The variances of all Lagrangian points are stored in one-dimensional arrays to ensure the coalescing and aligned access pattern to the global memory. The code of the MFDM on CPU uses a sequential structure similar to the Lagrangian mesh centric arrangement. The fluid field macroscopic variables like velocities and forces should be transferred from GPU to CPU before IBM part and the modified velocities and forces should be copied back to GPU when IBM part finishes.

All the simulations are performed in parallel on an Nvidia Tesla GPU K40c ${ }_{525}$ (compute capability 3.5) and sequentially on a single core of CPU (Intel(R) Xeon(R) CPU E5-2640 v3 @ 2.60GHz) with the same computational domain. Fig. 21 displays the ratio of the computational time consumed by the MDFM part on GPU and its sequential counterpart on CPU to quantify the computational performance. By increasing the number of Lagrangian points, the speedup of the MDFM part grows quickly and exceeds 20. A maximum speed-up of 23.7 is achieved when dealing with 9500 Lagrangian points with 64 threads per block. it is important to note that if all the cores on the CPU were utilised using a shared memory programming strategy (OpenMP) - and even if we assume a linear speed-up on all available 8 cores - the GPU parallel code still would show a significant advantage over the CPU parallel code. In addition, a linear speed-up on the CPU is also not expected due to the similar need to deal with a similar race condition in the GPU parallel code.

Moreover, an optimisation strategy that uses shared memory could significantly improve efficiency by avoiding the repeated read to the device memory 540 in the interpolation procedure. In addition, the Lagrangian points near a lattice could be found efficiently, the atomic operations could be avoided in the distribution procedure by using the Eulerian mesh centric arrangement when 
utilizing the great number of threads on GPU. Thus, there are still plenty of opportunities to boost the computational efficiency and resolve a large number of Lagrangian points with reasonable performance.

\section{References}

[1] Aidun, C.K., Ding, E.J.. Dynamics of particle sedimentation in a vertical channel: Period-doubling bifurcation and chaotic state. Physics of Fluids 2003;15(6):1612-1621.

[2] Verjus, R., Guillou, S., Ezersky, A., Angilella, J.R.. Chaotic sedimentation of particle pairs in a vertical channel at low Reynolds number: Multiple states and routes to chaos. Physics of Fluids 2016;28(12).

[3] Aidun, C.K., Clausen, J.R.. Lattice-Boltzmann Method for Complex Flows. Annual Review of Fluid Mechanics 2010;42(1):439-472.

[4] Chen, S., Doolen, G.D.. Lattice boltzmann method for fluid flows. Annual review of fluid mechanics 1998;30(1):329-364.

[5] Ladd, A.J.C.. Numerical simulations of particulate suspensions via a discretized Boltzmann equation. Part 1. Theoretical foundation. Journal of Fluid Mechanics 1994;271:285.

[6] Ladd, A.J.C.. Numerical simulations of particulate suspensions via a discretized Boltzmann equation. Part 2. Numerical results. Journal of Fluid Mechanics 1994;271:311.

[7] Feng, J., Hu, H.H., Joseph, D.D.. Direct simulation of initial value problems for the motion of solid bodies in a newtonian fluid part 1. sedimentation. Journal of Fluid Mechanics 1994;261:95-134.

[8] Xiao, Y., Yang, L., Zhou, Y., Wei, Y., Wang, N.. Dominant parameters affecting the reliability of TBCs on a gas turbine blade during erosion by a particle-laden hot gas stream. Wear 2017; 
[9] Geier, M., Greiner, A., Korvink, J.G.. Cascaded digital lattice Boltzmann automata for high Reynolds number flow. Physical Review E 2006;73(6):110.

[10] Chen, H., Teixeira, C., Molvig, K.. Digital physics approach to computational fluid dynamics: some basic theoretical features. International Journal of Modern Physics C 1997;8(04):675-684.

[11] Latt, J., Chopard, B.. Lattice boltzmann method with regularized precollision distribution functions. Mathematics and Computers in Simulation 2006;72(2-6):165-168.

[12] Ning, Y., Premnath, K.N., Patil, D.V.. Numerical study of the properties of the central moment lattice boltzmann method. International Journal for Numerical Methods in Fluids 2016;82(2):59-90.

[13] Premnath, K.N., Banerjee, S.. Incorporating forcing terms in cascaded lattice boltzmann approach by method of central moments. Physical Review E 2009;80(3):036702.

[14] Nie, X.B., Shan, X., Chen, H.. Galilean invariance of lattice boltzmann models. Europhysics Letters 2008;81:34005.

[15] Chen, H., Gopalakrishnan, P., Zhang, R.. Recovery of galilean invariance in thermal lattice boltzmann models for arbitrary prandtl number. International Journal of Modern Physics C 2014;25:1450046.

[16] De Rosis, A.. Alternative formulation to incorporate forcing terms in a lattice boltzmann scheme with central moments. Physical Review E 2017;95(2):023311.

[17] De Rosis, A.. Central-moments-based lattice boltzmann schemes with force-enriched equilibria. EPL (Europhysics Letters) 2017;117(3):34003.

[18] Hajabdollahi, F., Premnath, K.. Improving the low mach number steady state convergence of the cascaded lattice boltzmann method by preconditioning. Computers \& Mathematics with Applications 2017;. 
[19] De Rosis, A.. A central moments-based lattice boltzmann scheme for shallow water equations. Computer Methods in Applied Mechanics and Engineering 2017;319:379-392.

[20] Geier, M., Pasquali, A., Schönherr, M.. Parametrization of the cumulant lattice boltzmann method for fourth order accurate diffusion part i: Derivation and validation. Journal of Computational Physics 2017;348:862-888.

[21] Geier, M., Pasquali, A., Schönherr, M.. Parametrization of the cumulant lattice boltzmann method for fourth order accurate diffusion part ii: Application to flow around a sphere at drag crisis. Journal of Computational Physics 2017;348:889-898.

[22] Haeri, S., Shrimpton, J.S.. A mesoscopic description of polydispersed particle laden turbulent flows. Progress in Energy and Combustion Science 2011;37:716-740.

[23] Haeri, S., Shrimpton, J.S.. On the application of immersed boundary, fictitious domain and body-conformal mesh methods to many particle multiphase flows. International Journal of Multiphase Flow 2012;40:38-55.

[24] Haeri, S., Shrimpton, J.S.. A new implicit fictitious domain method for the simulation of flow in complex geometries with heat transfer. Journal of Computational Physics 2013;237:21-45.

[25] Haeri, S., Shrimpton, J.S.. Fully resolved simulation of particle deposition and heat transfer in a differentially heated cavity. International Journal of Heat and Fluid Flow 2014;50:1-15.

[26] Peskin, C.S.. Numerical analysis of blood flow in the heart. Journal of Computational Physics 1977;25(3):220-252.

[27] De Rosis, A., Lévêque, E.. Central-moment lattice Boltzmann schemes with fixed and moving immersed boundaries. Computers and Mathematics with Applications 2016;72(6):1616-1628. 
[28] Wang, Z., Fan, J., Luo, K.. Combined multi-direct forcing and immersed boundary method for simulating flows with moving particles. International Journal of Multiphase Flow 2008;34(3):283-302.

[29] Suzuki, K., Inamuro, T.. Effect of internal mass in the simulation of a moving body by the immersed boundary method. Computers and Fluids 2011;49(1):173-187.

[30] Suzuki, K., Okada, I., Yoshino, M.. Accuracy of the laminar boundary layer on a flat plate by an immersed boundary-lattice Boltzmann method. Journal of Fluid Science and Technology 2016;11(3):16-00488.

[31] Minami, K., Suzuki, K., Inamuro, T.. Free flight simulations of a dragonfly-like flapping wing-body model using the immersed boundarylattice Boltzmann method. Fluid Dynamics Research 2015;47(1):15505.

[32] Haeri, S.. Optimisation of blade type spreaders for powder bed preparation in additive manufacturing using dem simulations. Powder Technology 2017;321:94-104.

[33] Haeri, S., Wang, Y., Ghita, G., Sun, J.. Discrete element simulation and experimental study of powder spreading process in additive manufacturing. Powder Technology 2017;45-54:306.

[34] Zhang, P., Galindo-Torres, S.A., Tang, H., Jin, G., Scheuermann, A., Li, L.. An efficient Discrete Element Lattice Boltzmann model for simulation of particle-fluid, particle-particle interactions. Computers and Fluids 2017;147:63-71.

[35] Jayaweera, K.O.L.F., Mason, B.J.. The behaviour of freely falling cylinders and cones in a viscous fluid. J Fluid Mech 1965;22(04):709.

[36] Qian, Y.H., D'Humières, D., Lallemand, P.. Lattice BGK Models for Navier-Stokes Equation. EPL (Europhysics Letters) 1992;17:479-484. 
[37] Wu, J., Shu, C.. Implicit velocity correction-based immersed boundarylattice Boltzmann method and its applications. Journal of Computational Physics 2009;228(6):1963-1979.

[38] Guo, Z., Zheng, C., Shi, B.. Discrete lattice effects on the forcing term in the lattice Boltzmann method. Physical Review E - Statistical, Nonlinear, and Soft Matter Physics 2002;65(4):1-6.

[39] Haeri, S., Shrimpton, J.. A correlation for the calculation of the local nusselt number around circular cylinders in the range $10 \leq$ re $\leq 250$ and 0.1 $\leq \operatorname{pr} \leq 40$. International Journal of Heat and Mass Transfer 2013;59:219229.

[40] Suzuki, K., Inamuro, T.. A higher-order immersed boundary-lattice Boltzmann method using a smooth velocity field near boundaries. Computers and Fluids 2013;76:105-115.

[41] Feng, Z.G., Michaelides, E.E.. Robust treatment of no-slip boundary condition and velocity updating for the lattice-Boltzmann simulation of particulate flows. Computers and Fluids 2009;38(2):370-381.

[42] Luding, S.. Introduction to discrete element methods: Basic of contact force models and how to perform the micro-macro transition to continuum theory. European Journal of Environmental and Civil Engineering 2008;8189:785-826.

[43] Vasseur, P., Cox, R.G.. The lateral migration of spherical particles sedimenting in a stagnant bounded fluid. Journal of Fluid Mechanics 1977;80(03):561.

[44] Hu, H., Fortes, a., Joseph, D.. Experiments and direct simulations of fluid particle motions. Intern Video J Engng Res 1993;2:17-24.

[45] Fortes, A.F., Joseph, D.D., Lundgren, T.S.. Nonlinear mechanics of fluidization of beds of spherical particles. Journal of Fluid Mechanics 1987;177:467-483. 
[46] Ardekani, A., Rangel, R.. Numerical investigation of particle-particle and particle-wall collisions in a viscous fluid. Journal of fluid mechanics 2008;596:437-466.

[47] Wu, J., Cheng, Y., Zhou, W., Zhang, C., Diao, W.. Gpu acceleration of fsi simulations by the immersed boundary-lattice boltzmann coupling scheme. Computers \& Mathematics with Applications 2016; 
Table 1: The $R e_{t}$ of a pair of particles sedimentation

\begin{tabular}{|c|c|c|c|c|c|c|c|c|c|}
\hline \multicolumn{2}{|c|}{$F$} & 130 & 150 & 160 & 300 & 350 & 400 & 450 & 480 \\
\hline \multirow{2}{*}{ States } & With rotation & Oblique & Oblique & Period-d & DKT & DKT & DKT & Quasi-p & Quasi-p \\
\cline { 2 - 11 } & Without rotation & Oblique & Oblique & Oblique & Oblique & Horizon & Horizon & Horizon & Horizon \\
\hline \multirow{2}{*}{$R e_{t}$} & Without rotation & 2.08 & 2.43 & 2.59 & 3.5 & 4.35 & 4.75 & 5.14 & 5.45 \\
\cline { 2 - 11 } & With rotation & 2.24 & 2.75 & 4.15 & 8.23 & 9.25 & 10.16 & 5.2 & 5.61 \\
\hline \multicolumn{2}{|c|}{ Ratio(\%) } & 92.86 & 88.36 & 62.41 & 42.53 & 47.03 & 46.75 & 98.85 & 97.15 \\
\hline
\end{tabular}




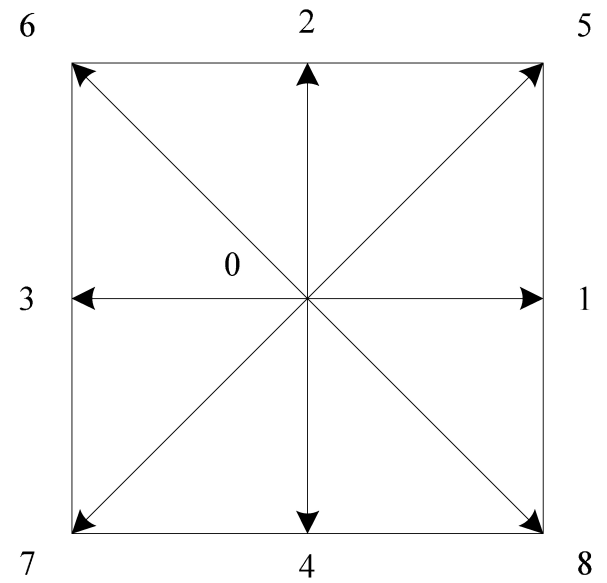

Figure 1: Lattice pattern: D2Q9 


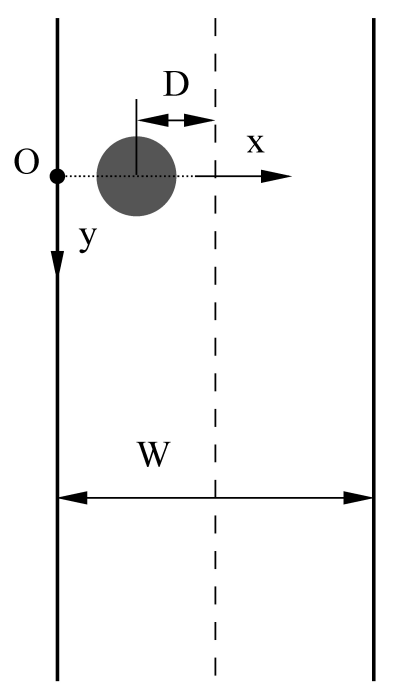

Figure 2: Initial geometry for the sedimentation of a single particle. 


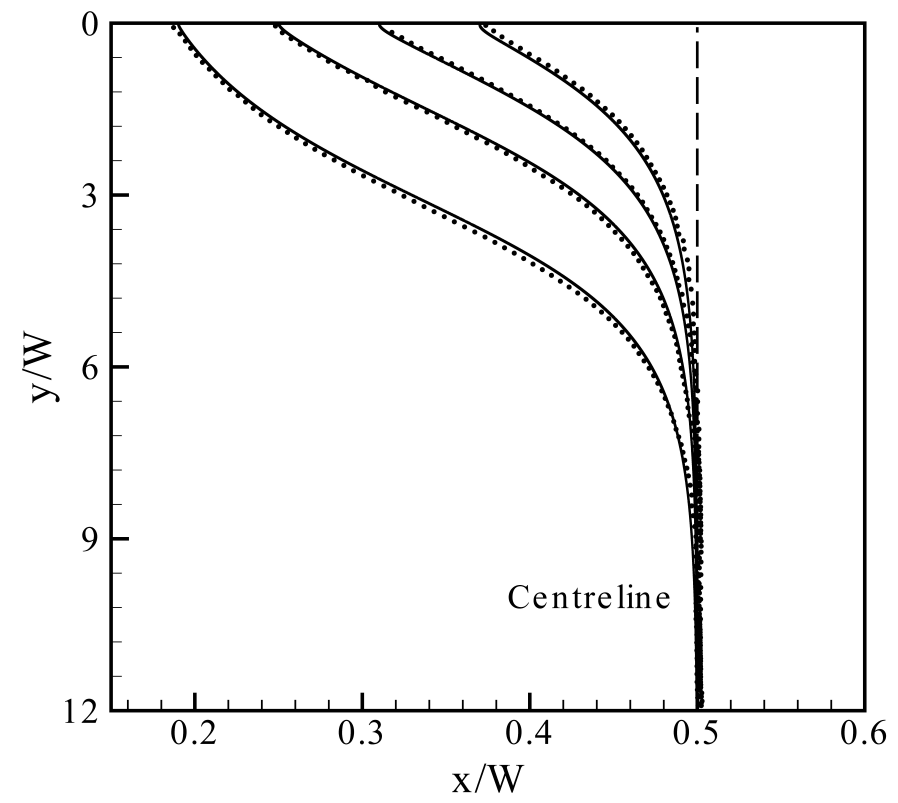

Figure 3: The settling trajectories of particles released from different initial positions, $x / W=$ $(0.19,0.25,0.31,0.37)$. The present results: - with $F=12$ and $R e_{t}=0.525 ;$ results from Feng et al. [7]: $\cdots$, with $\operatorname{Re}_{t}=0.522$. The centreline $x / W=0.5$ is marked with a long dash line. 


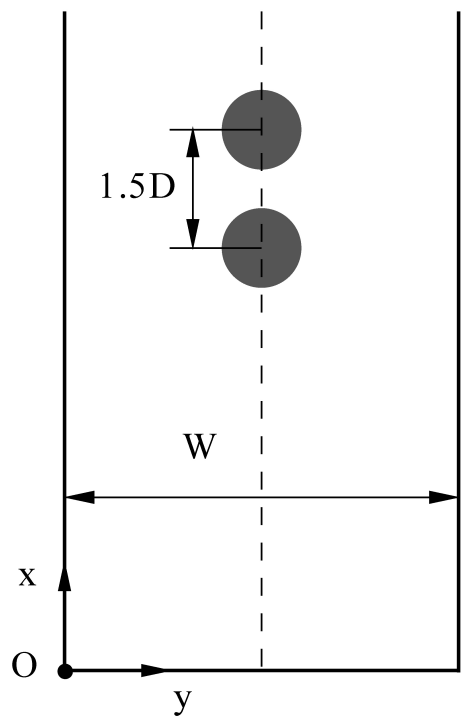

Figure 4: Initial geometry for the DKT of two particles. 


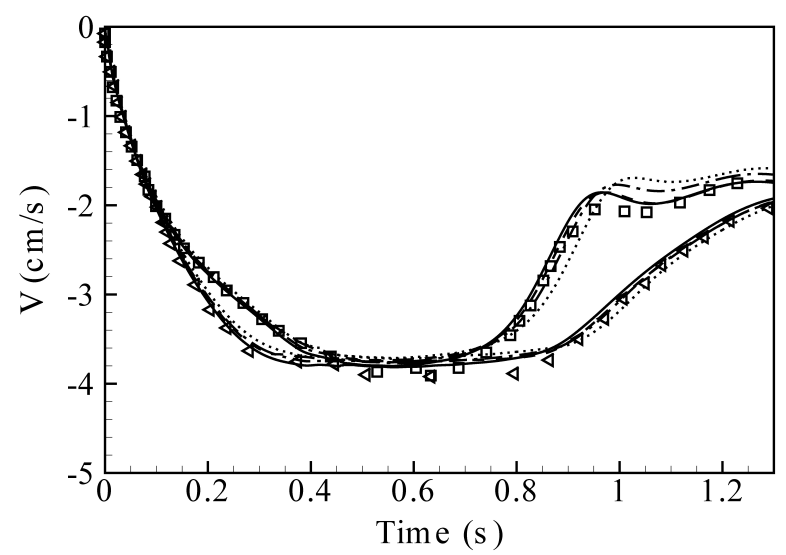

(a)

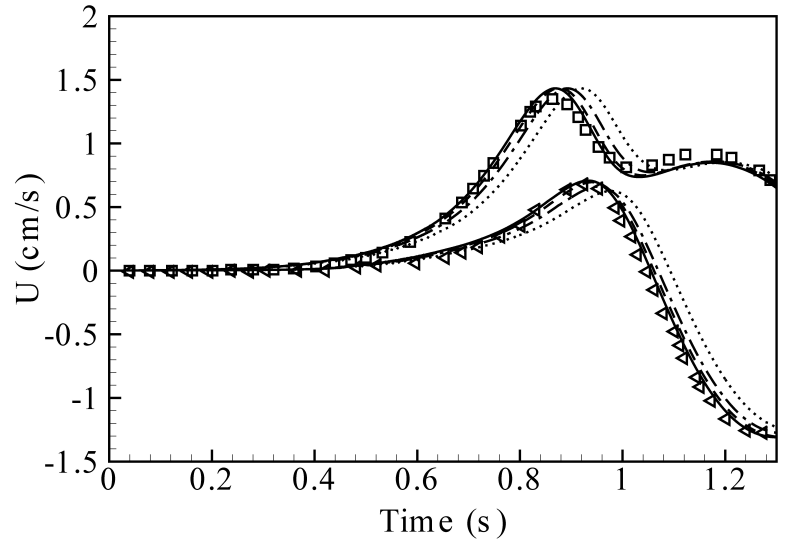

(b)

Figure 5: Linear velocity of the two particles. Leading particle and trailing particle are marked with solid line and dashed line, respectively. Grid independence of the collision strategy is tested, $D=15 \delta x$ : dotted line; $D=20 \delta x$ : dash dot line; $D=25 \delta x$ : dashed line; $D=30 \delta x$ : solid line. Results from Ardekani et al. 46]: leading particle $\triangle$; trailing particle $\square$. 

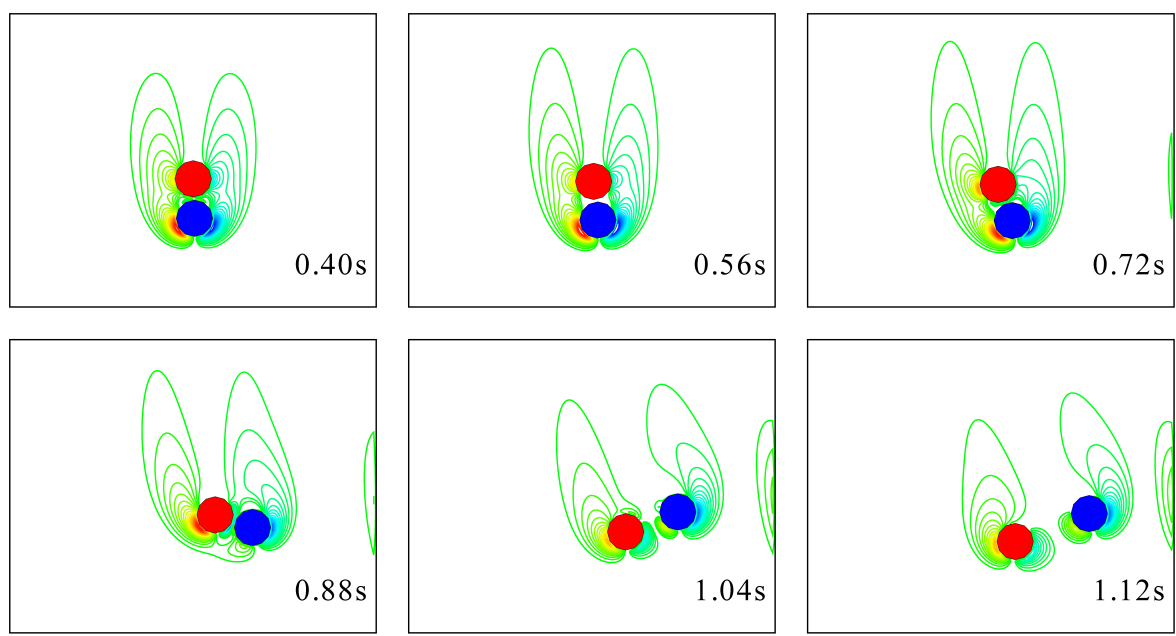

Figure 6: Vorticity contours at five time instants $(0.40 \mathrm{~s}, 0.56 \mathrm{~s}, 0.72 \mathrm{~s}, 0.88 \mathrm{~s}, 1.04 \mathrm{~s}$ and $1.12 \mathrm{~s})$ during DKT. 


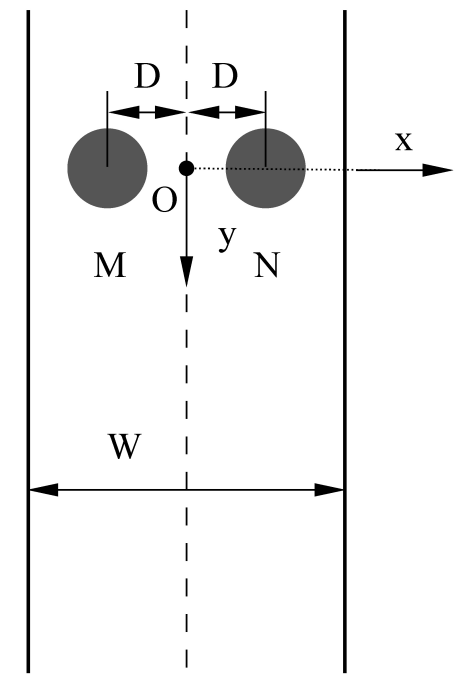

Figure 7: Initial geometry for the sedimentation of a pair of particles. 


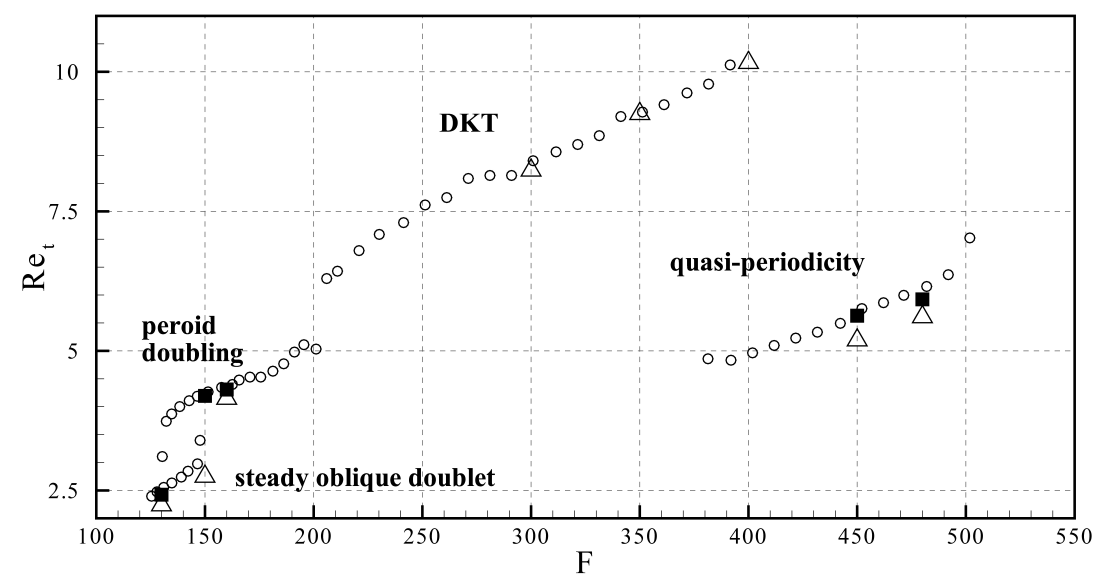

Figure 8: Global diagram: the terminal Reynolds number $R e_{t}$ versus the non-dimensional driving force $F$ and the corresponding principal movement states. The present results: $\Delta$, $D=25 \delta x ; \mathbf{\square}, D=50 \delta x$. The results from Verjus et al. [2]: $\mathrm{\text {. }}$ 

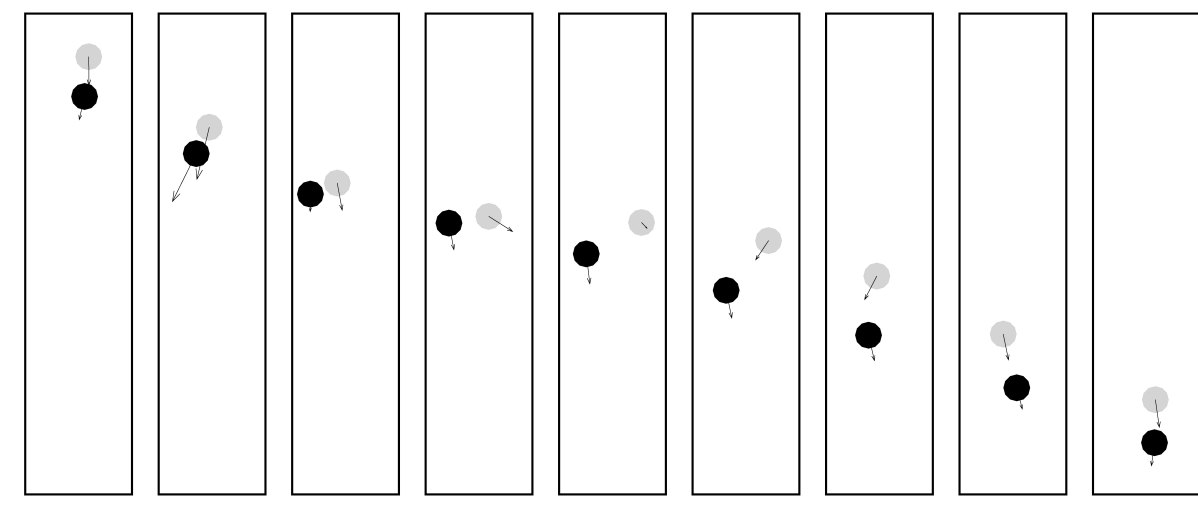

Figure 9: The DKT of particles $(F=300)$ with velocity represented by arrows. 


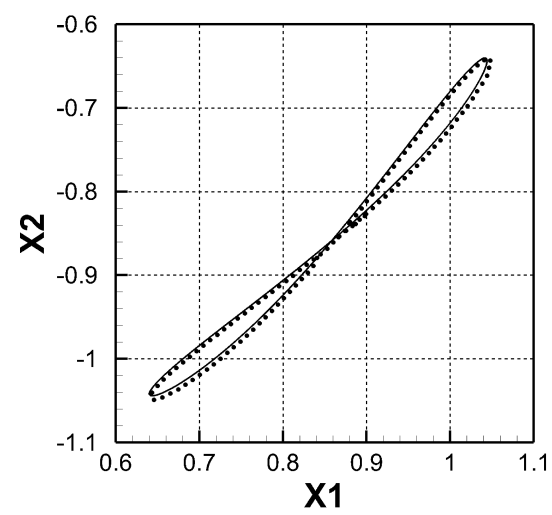

Figure 10: The phase portrait of $F=480$. The present work with $D=50 \delta x$ : - ; the results from Verjus et al. 2]: $\cdots$; X1 and X2 are the non-dimensional lateral locations of Particle 1 and Particle 2, respectively. 


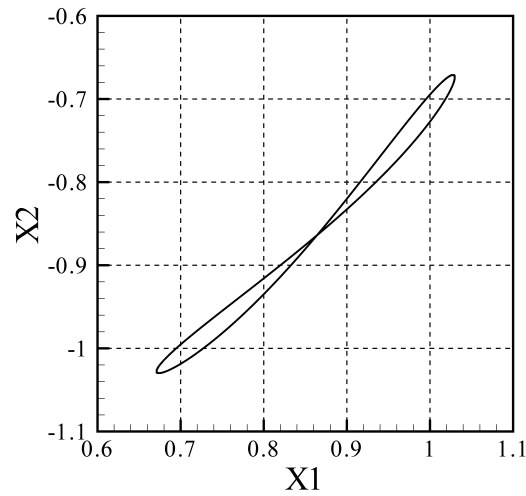

(a)

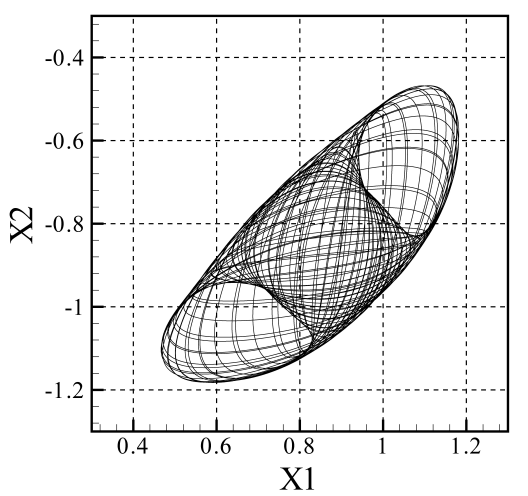

(b)

Figure 11: The phase portrait of $F=450$ and $F=480$ of the present work with $D=25 \delta x$, with X1 and X2 the lateral non-dimensional location of Particle 1 and Particle 2, respectively. 


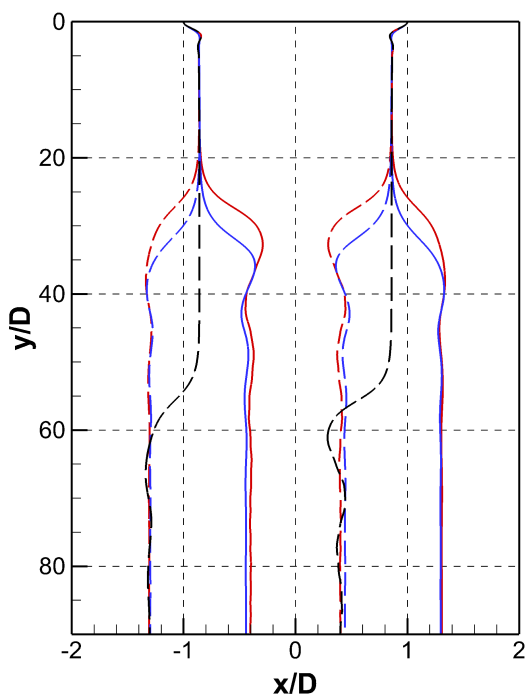

(a) $F=150$, oblique doublet

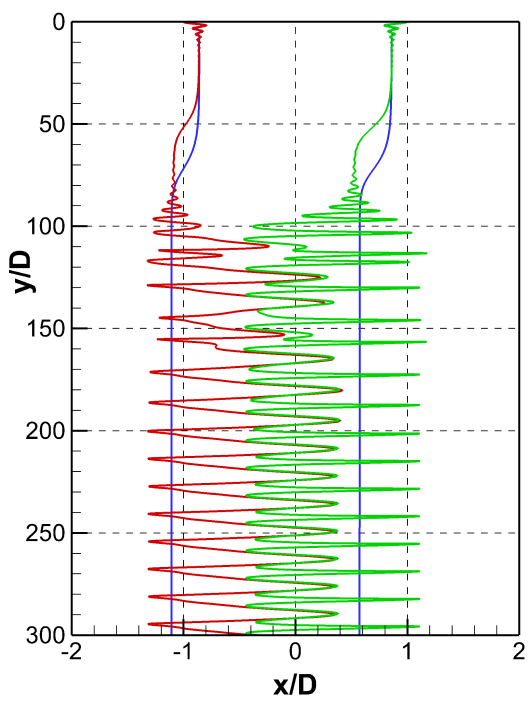

(c) $F=300$, DKT

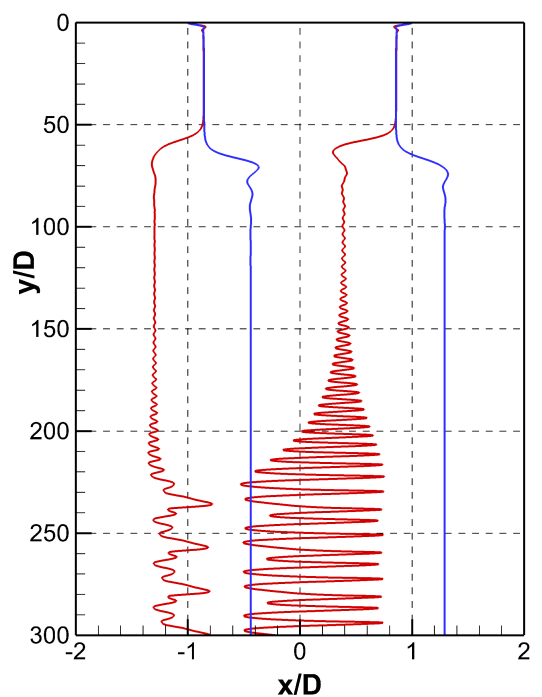

(b) $F=160$, period doubling

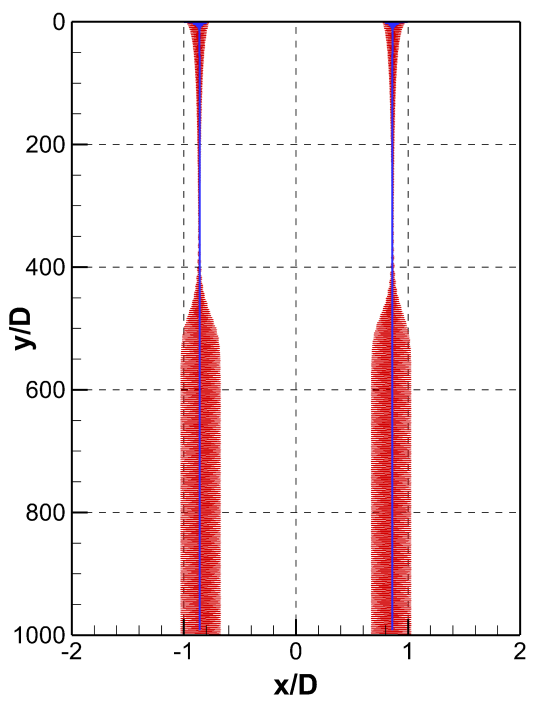

(d) $F=450$, quasi-periodic 


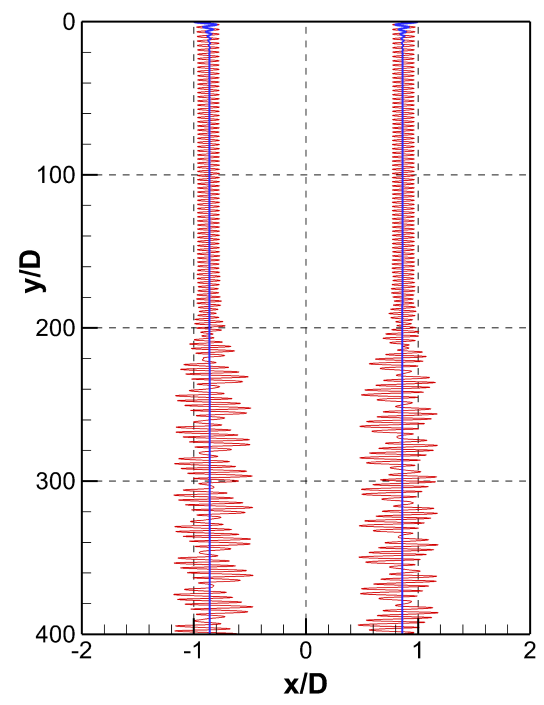

(e) $F=480$, quasi-periodic

Figure 12: The trajectories of the particle pairs with different principal movement states, The particles with natural rotation: - (Particle 2 of $F=300$ : - - ; The particles without the rotational degree-of-freedom: — The particles $(F=150)$ without initial disturbance and with natural rotation: - - -; The particles $(F=150)$ with an opposite disturbance and natural rotation: - - - The particles $(F=150)$ with an opposite disturbance and without rotational degree-of-freedom: - - - 

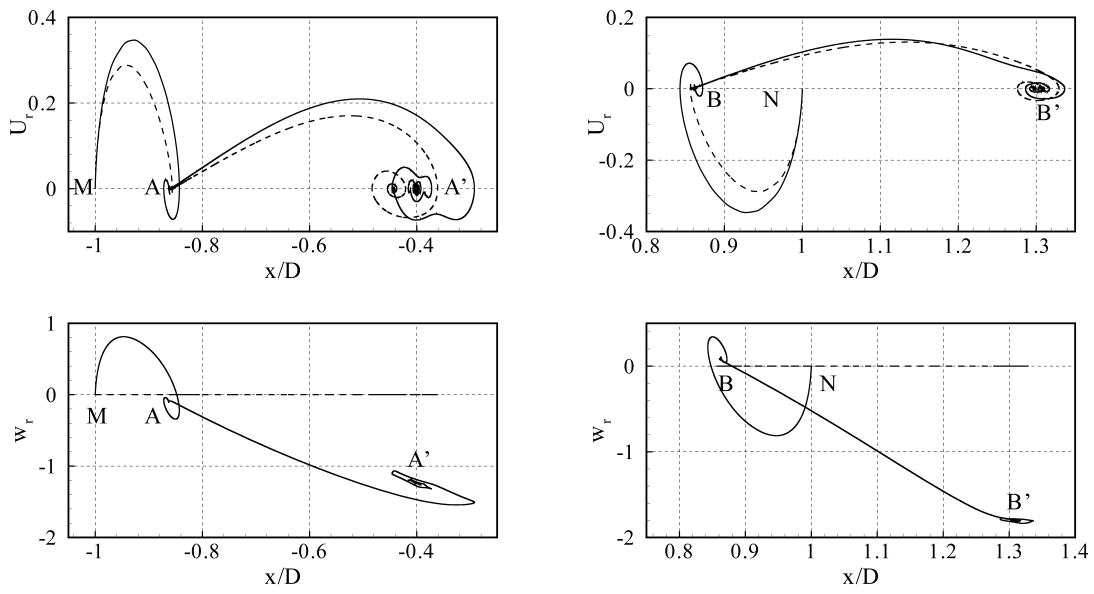

(a) Particle 1

(b) Particle 2

Figure 13: The non-dimensional lateral and angular velocity of particles with $F=150$, solid line: with rotational degree-of-freedom; dashed line: without rotational degree-of-freedom. (Positive $\omega_{r}$ : counter-clockwise; negative $\omega_{r}$ : clockwise.) 


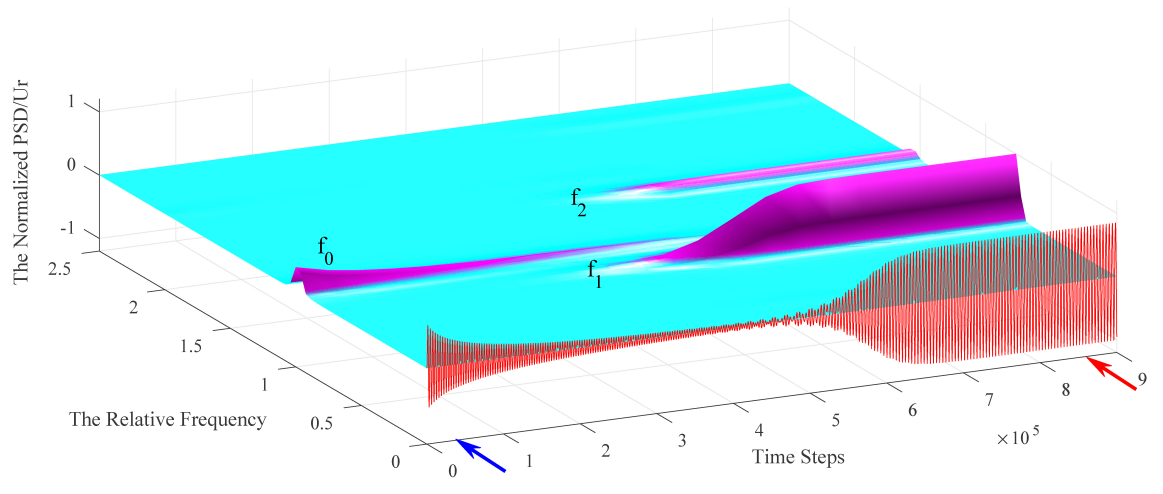

(a)

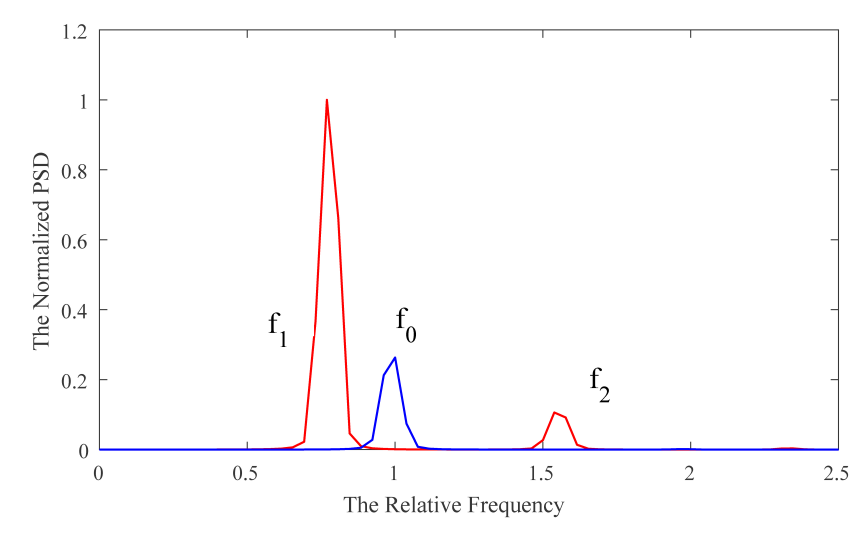

(b)

Figure 14: The time-frequency analysis to the lateral velocity of Particle $1(F=450)$ (a) The oscillation of $U_{r}$ along the time steps and the corresponding spectrogram. (b) The spectrogram of time steps marked with arrows in Fig $14 \mathrm{a}$ 


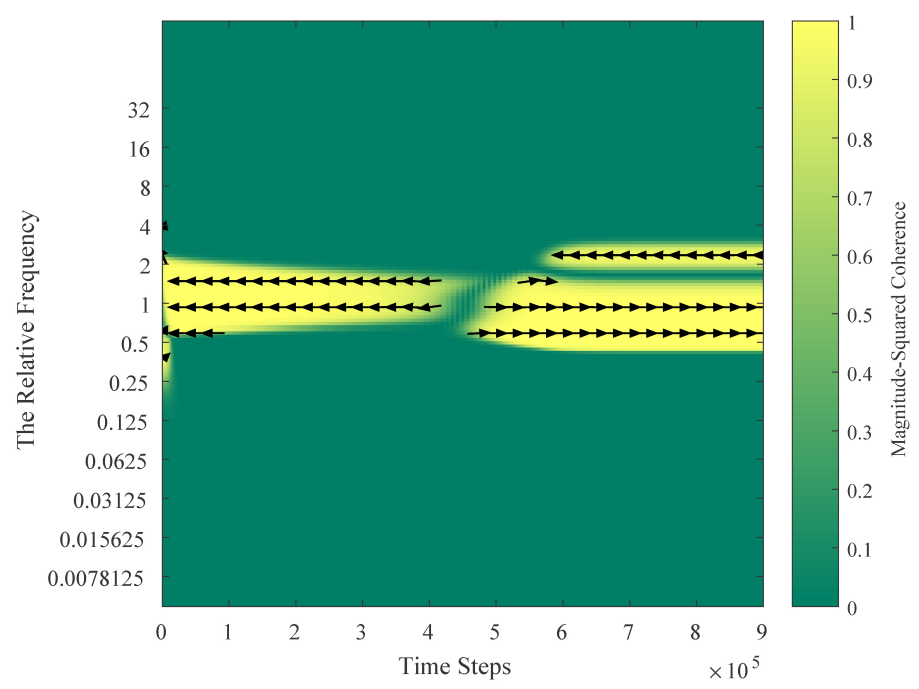

(a)

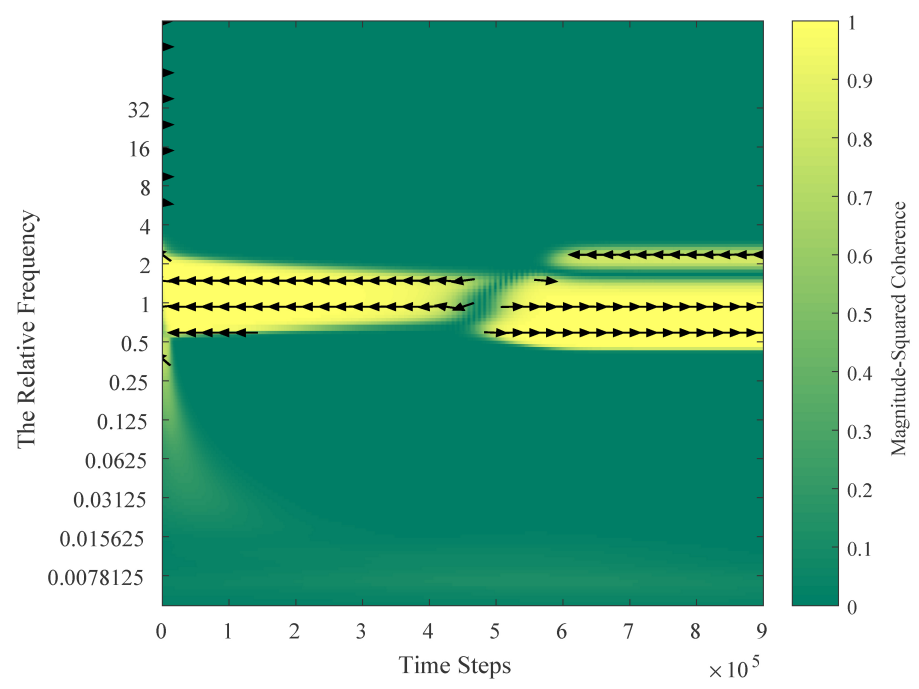

(b)

Figure 15: The coherence of the nonstationary movement of Particle $1 \& 2(F=450)$ with time and frequency variation. (a) The lateral velocities of Particle $1 \& 2$; (b) The angular velocities of Particle $1 \& 2$. 


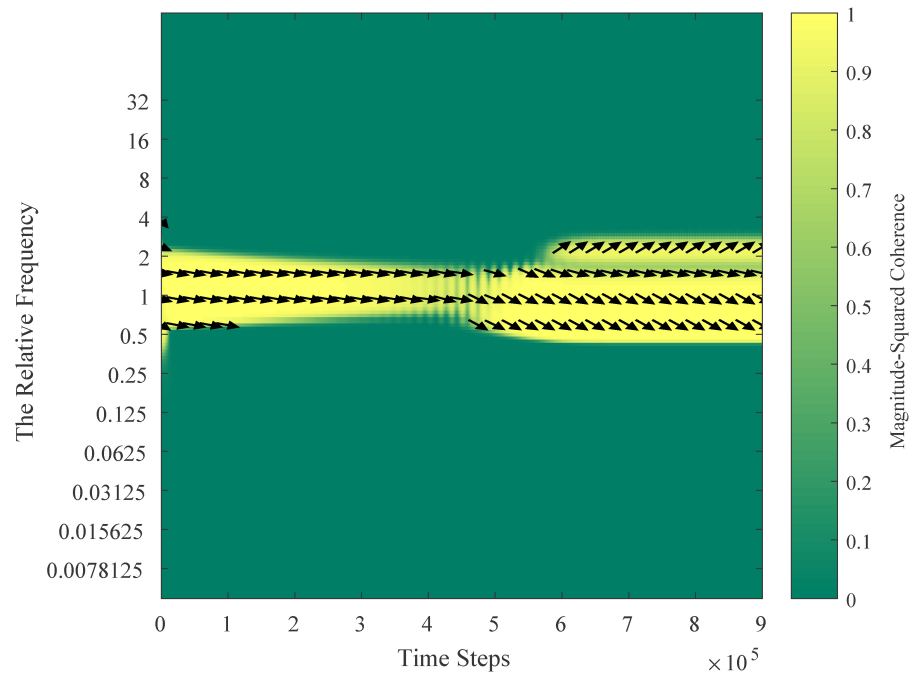

Figure 16: The coherence of the lateral and the angular velocities of Particle $1(F=450)$ with frequency and time variation. 


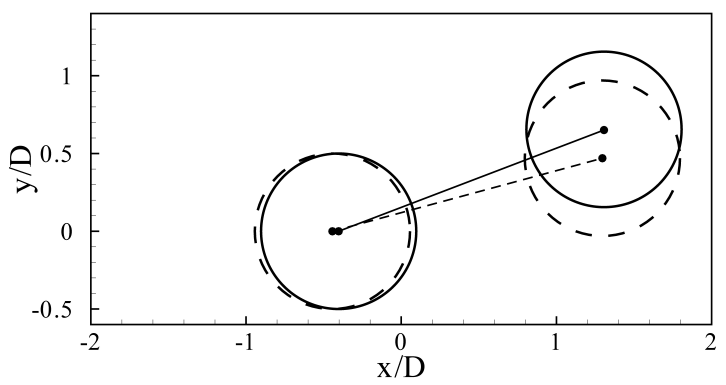

Figure 17: The oblique doublet structure of particles $(F=150)$, with natural rotation: without the rotational degree-of-freedom: - - -. 


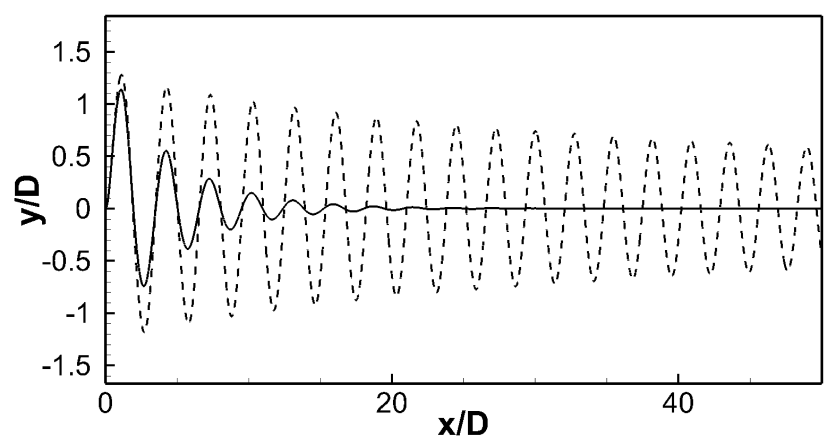

Figure 18: The damping oscillation of Particle $1(F=450)$ after released from rest, with natural rotation: - - -; without the rotational degree-of-freedom: - 


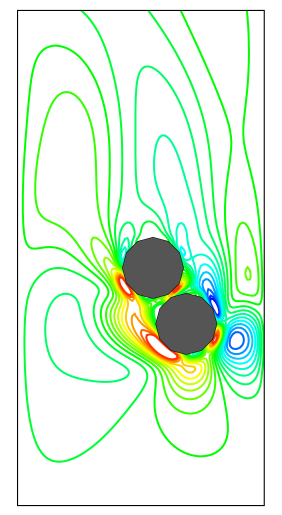

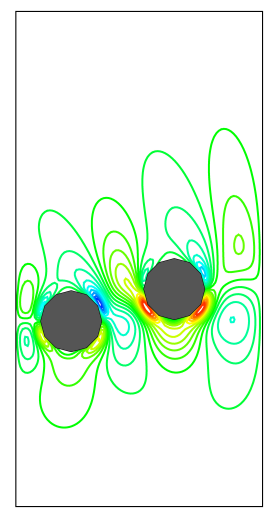

(a)
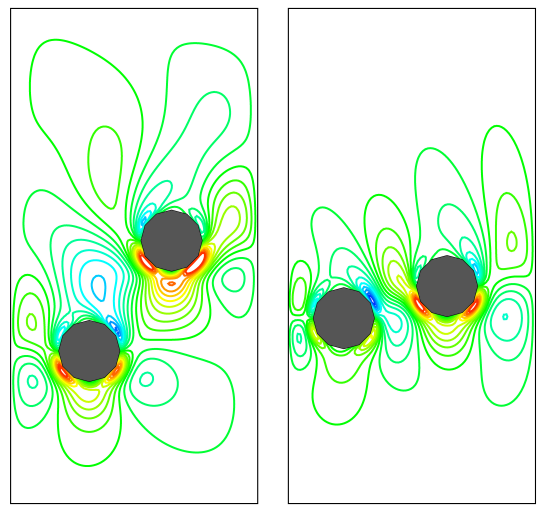

(b)

Figure 19: The vorticity coutour of $F=300$, (a) time $t_{1}$, left: with natural rotation; right: without rotational degree-of-freedom; (b) time $t_{2}$, left: with natural rotation; right: without rotational degree-of-freedom. 


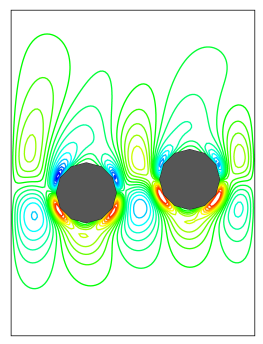

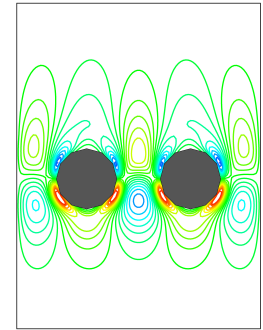

(a)
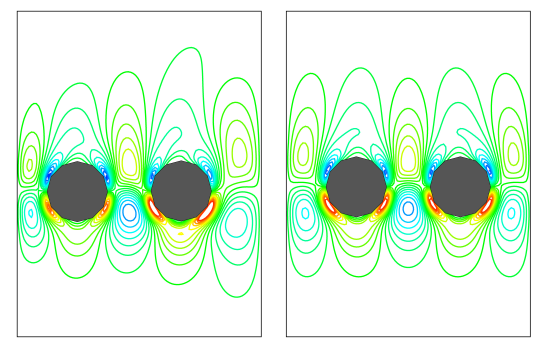

(b)

Figure 20: The vorticity contour of $F=450$, (a) time $t_{1}$, left: with natural rotation; right: without rotational degree-of-freedom; (b) time $t_{2}$, left: with natural rotation; right: without rotational degree-of-freedom. 


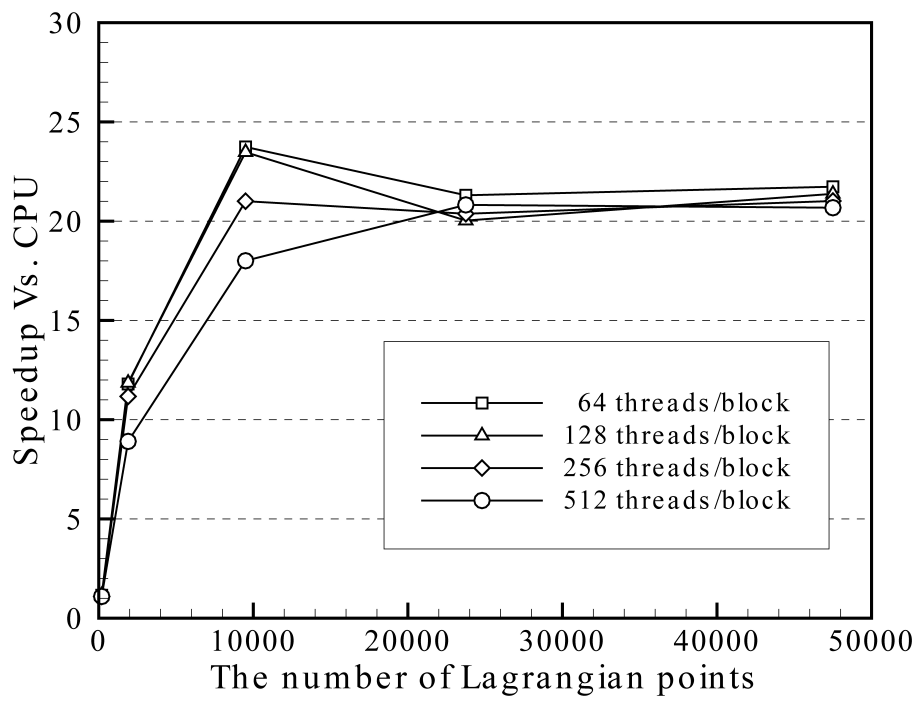

Figure 21: The speedup of performance of MDFM (IBM model) part on a GPU vs. its sequential counterpart on a CPU processor

when dealing with different numbers of Lagrangian points. 